Arch. Vet. Scienc. 3(1):73-81, 1998

Printed in Brazil

\title{
INFLUÊNCIA DA TEMPERATURA E DO pH NAS PROPRIEDADES CINÉTICAS DA LACTATO DESIDROGENASE DO MÚSCULO EPAXIAL DE Prochilodus scropha e Notothenia neglecta*.
}

\section{CLEONI SANTOS CARVALHO ${ }^{1}$; RUBENS ROSA ${ }^{2}$; KIKUE T. SASSAKI ${ }^{3}$; METRY BACILA}

\author{
Estação Antártica Brasileira Comandante Ferraz, Ilha do Rei Jorge, Shetlands do Sul. \\ ${ }^{1}$ Laboratório de Piscicultura da Universidade Federal do Paraná, CEP 80.000-970, Curitiba-Pr-Brasil. \\ ${ }^{2}$ Departamento de Medicina, Pontifícia Universidade Católica do Paraná - PUC-PR. ${ }^{3}$ Departamento de Ciências \\ Fisiológicas, Faculdade de Odontologia de Araçatuba, UNESP, Araçatuba, São Paulo.
}

\begin{abstract}
It has been carried out a comparative study on the kinetic properties of the lactate dehydrogenase (L-lactate $\mathrm{NAD}^{+}$oxidoreductase, E.C.1.1.1.27) purified from the epaxial muscle of the tropical fish Prochilodus scropha and the Antarctic fish Notothenia neglecta. The following parameters were studied: a. Effect of pH; effect of temperature and values for energy of activation; effect of substrate concentration and the effect of temperature on the $\mathrm{Km}$ values.
\end{abstract}

KeyWords: Lactate dehydrogenase; Prochilodus scropha; Notothenia neglecta.

RESUMO - Foi levado a efeito um estudo comparativo das propriedades cinéticas da lactico desidrogenase (Llactato $\mathrm{NAD}^{+}$oxidorreductase, E.C.1.1.1.27) purificada do músuclo epaxial do peixe tropical Prochilodus scropha e do peixe antártico Notothenia neglecta. Os seguintes parâmetros foram estudados: a. Efeito do pH; efeito da temperatura e valores para a energia de ativação; efeito da concentração de substrato e o efeito da temperatura sobre os valores de Km.

Palavras-Chave: Lactato desidrogenase; Prochilodus scropha; Notothenia neglecta.

\section{Introdução}

A láctico desidrogenase (LDH) (L-Lactato: NADH oxidorredutase, E.C.1.1.1.27), é de fundamental importância em processos envolvendo o metabolismo glicídico. Essa enzima catalisa a transformação reversível do lactato para piruvato, com suporte coenzimático do sistema NAD+/NADH $+\mathrm{H}^{+}$. É essencial para o suprimento de $\mathrm{NAD}+$ para a reação da gliceraldeído-fosfato desidrogenase e, desta forma, direcionar a finalização aeróbica ou anaeróbica da via glicolítica, frente às necessidades metabólicas do tecido.

A LDH é um tetrâmero de PM 140.000. Cada subunidade liga-se a uma molécula de coenzima e reage independentemente com o substrato (HECK,1969). Dois tipos de subunidades estão presentes na proteína oligomérica de LDH e o padrão isoenzimático está relacionado com o tipo de metabolismo de cada tecido: o tipo $\mathrm{H}$ (coração) e o tipo $\mathrm{M}$ (músculo), de acordo com os órgãos em que foram obtidos (DAWSON et al., 1964). O tipo $\mathrm{H}$ (ou tipo B) predomina em tecidos de metabolismo aeróbico, tal como o músculo cardíaco. É composta de 4 subunidades $\left(\mathrm{H}_{4}\right)$, sendo inibida por altas concentrações de piruvato (dentro de uma taxa fisiológica) e com baixo $K_{M}$ para este

\footnotetext{
*Com base na Tese de Mestrado de Cleoni Santos Carvalho, Curso de Pós-Graduação em Ciências Veterinárias, UFPR. Com auxílio da Programa Antártico Brasileiro - PROANTARCNPq.

${ }^{1}$ Bolsista de Pós-Graduação do CNPq
}

substrato. O tipo $\mathrm{M}$ (ou tipo A) predomina em tecidos como o músculo esquelético, o qual deriva grande parte de sua energia da glicólise anaeróbica. A isoenzima do músculo esquelético está adaptada para converter altas concentrações de piruvato a lactato apresentando alto $K_{M}$ e Vmax elevado para o piruvato (SIDELL e BELAND, 1980).

$\mathrm{O}$ tipo $\mathrm{H}_{4}$ oxida lactato a piruvato, sendo este utilizado como combustível pelo coração. O metabolismo aeróbico do coração permite que ele encaminhe o piruvato para o ciclo do ácido cítrico. $\mathrm{O}$ tipo $\mathrm{M}_{4}$ opera na direção oposta, convertendo piruvato em lactato para permitir que a glicólise se processe em condições anaeróbicas. As interconversões entre piruvato e lactato são facilitadas por diferenças nas propriedades catalíticas das isoenzimas de LDH em diferentes tecidos.

Em mamíferos e aves, e em alguns peixes teleósteos, existe um terceiro tipo de $\mathrm{LDH}$, denominada de $\mathrm{LDH} \mathrm{X}$ ou isoenzima C. Em peixes, ela é encontrada no fígado (REHSE e DAVIDSON, 1985), no cérebro e no olho (FITCH, 1988, COPPES et al., 1992) e em ratos, no espermatócito (SPIELMANN et al., 1973).

As isoenzimas $\mathrm{H}$ e $\mathrm{M}$ são similares no peso molecular mas diferem grandemente em propriedades físicas, químicas e imunológicas, como também nas propriedades catalíticas e em sua distribuição nos tecidos (VESELL, 1966; VESELL e YIELDING, 1966 e BAILEY e WILSON, 1968).

Com relação aos fatores físicos que


frequentemente variam no ambiente, a temperatura é importante do ponto de vista fisiológico e bioquímico. Segundo HOCHACHKA e SOMERO (1968); GEREZ DE BURGOS et al., (1973); NARITA e HORIUCHI (1979); FITCH (1988); KLYACHKO e OZERNYUK, (1994) e RODRIGUES et al., (1995) o $\mathrm{K}_{\mathrm{M}}$ para a LDH aumenta com a elevação da temperatura e tende a ser maior para a enzima de espécies adaptadas a altas temperaturas (YANCEY e SOMERO, 1978; TSUKUDA, 1975; SOMERO, 1981 e OZERNYUK et al., 1994).

Peixes da família Notothenidae são abundantes e amplamente distribuídos nos mares antárticos, sendo o grupo de peixes mais diversificado em relação à espécie, habitat e distribuição. Evidências sugerem que espécies de peixes antárticos tornaram-se isoladas pela convergência circumpolar Antártica durante o Período Terciário e têm evoluído independentemente de outros grupos de teleósteos sob pressão seletiva de glaciações sucessivas desde aquele tempo (De WITT, 1971).

O presente trabalho descreve algumas propriedades da LDH do músculo estriado branco de Notothenia neglecta e de curimbatá, Prochilodus scropha. Assim, o objetivo deste trabalho é o de analisar, a nível molecular, os mecanismos bioquímicos de adaptações biológicas experimentados por peixes antárticos em seu ecossistema antártico em comparação com a de peixes das regiões tropicais.

\section{Material e Métodos}

A LDH foi obtida e purificada de acordo com CARVALHO (1997) e CARVALHO et al. (1998) a partir de músculo estriado branco de exemplares de Prochilodus scropha, curimbatá, capturados no Centro de Cultivo de Peixes do IBAMA, Pirassununga, São Paulo. Peixes adultos foram levados para o laboratório do Centro de Cultivo, onde foram sacrificados por secção da coluna vertebral. A seguir, o tecido muscular estriado foi dissecado, lavado em solução fisiológica a $4^{0} \mathrm{C}$, armazenado e mantido a $4^{0} \mathrm{C}$, e transportado para o Instituto de Química da Universidade de São Paulo, em isopor contendo gelo seco. O material biológico de Notothenia neglecta foi obtido durante a Operação Verão da XIV Expedição Antártica Brasileira. Peixes adultos foram levados para o Laboratório da Estação, onde foram sacrificados por secção da coluna vertebral. A seguir, o tecido muscular estriado foi dissecado, lavado em solução fisiológica a $4^{0} \mathrm{C}$, armazenado e mantido a $4^{0} \mathrm{C}$, e transportado para os nossos laboratórios em isopor contendo gelo seco.

Frações de cerca de trinta gramas de músculo estriado congelado de Prochilodus scropha e de Notothenia neglecta foram lavadas em solução salina $0,9 \%$ e ressuspensas em tampão fosfato $33 \mathrm{mM}, \mathrm{pH}$ 6,5 , contendo $\mathrm{MgCl}_{2} 1 \mathrm{mM}$ e $\beta \mathrm{ME} 0,5 \mathrm{mM}$. Os tecidos foram homogeneizados em tampão fosfato $\mathrm{pH}$ 6,5, na proporção de 1:4 de tampão em homogeneizador do tipo Potter-Elvehjem. Os homogeinizados foram centrifugados em centrífuga refrigerada $\mathrm{K} 70 \mathrm{D}$, a $4^{0} \mathrm{C}$, a $10.000 \mathrm{rpm}$, durante 30 minutos. Os sedimentos foram desprezados e os sobrenadantes, denominados extrato bruto (EB), foram mantidos em temperatura aproximada de $0-4^{0} \mathrm{C}$ até as etapas seguintes de purificação.

Foi adicionado ao EB, para ambas as preparações, sulfato de amônio sólido, lentamente, sob agitação constante, até atingir 70\% de saturação. Após agitação de 10 minutos e repouso de uma hora, a suspensão foi centrifugada a $10.000 \mathrm{rpm}$ por 30 minutos, a $4^{0} \mathrm{C}$. O sedimento (EB 70\%) foi ressuspenso no mesmo tampão de homogeneização, em volume suficiente para tanto. O EB 70\% foi dialisado contra tampão fosfato $10 \mathrm{mM}, \mathrm{pH}$ 6,5, contendo $\beta \mathrm{ME} 1 \mathrm{mM}$ e glicerol $10 \%$, durante aproximadamente 15 horas, a $4^{0} \mathrm{C}$. O dialisado foi centrifugado a $10.000 \mathrm{rpm}$ durante 30 minutos, a $4^{\circ} \mathrm{C}$. $\mathrm{O}$ sedimento foi desprezado e o sobrenadante (D70\%) utilizado nas etapas seguintes.

Nos experimentos que se seguem indica-se como fração D70\% a preparação ativa de LDH obtida por precipitação a $70 \%$ de saturação de sulfato de amônio, ressuspensa em tampão de homogeneização e dialisada em tampão fosfato $10 \mathrm{mM}, \mathrm{pH} \quad 6,5$ a contendo $\beta \mathrm{ME} 1 \mathrm{mM}$ e glicerol $10 \%$.

A atividade da $\mathrm{LDH}$ foi medida em meio contendo $\mathrm{NADH}+\mathrm{H}^{+}$0,14 $\mathrm{mM}$ e piruvato de sódio $1 \mathrm{mM}$, em

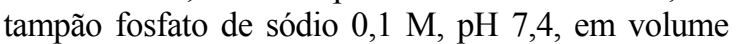
final de 1,0 ml, segundo BERGMEYER e BERN, 1974. A velocidade de oxidação do $\mathrm{NADH}+\mathrm{H}^{+}$foi acompanhada em espectrofotômetro BECKMAN DU 640, em comprimento de onda de $340 \mathrm{~nm}$ e à temperatura de $25^{\circ} \mathrm{C}$ para Prochilodus scropha e entre $8,0 \pm 2,0^{\circ} \mathrm{C}$ para Notothenia neglecta.

Uma unidade (UI) de LDH é definida como a quantidade de enzima que catalisa a transformação de 1 micromol de piruvato ou de lactato por minuto, nas condições de ensaio especificadas. A atividade específica é expressa em UI/mg de proteína.

A concentração de proteína foi determinada pelo método de LOWRY et al., (1951), utilizando-se como padrão protéico a albumina sérica bovina.

Substratos, coenzimas, lactato desidrogenase e outros produtos químicos foram obtidos da Sigma Chemical Co., St. Louis, MO, USA e produtos inorgânicos utilizados foram todos pró-análise.

Para verificar o efeito do $\mathrm{pH}$, a atividade da $\mathrm{LDH}$ da fração D70\% foi medida em meio de reação já descrito, porém foram utilizadas, para tanto, diferentes concentrações hidrogeniônicas: tampão citrato-fosfato 0,1 M pH 3.6, 4.6, 5.0, 5.6, 6.0, 7.0; tampão fosfato 0,2 M pH 7.8, 8.0, 8.5; tampão TRIS-HCl 0,2 M pH 8.6 e 9.0. A velocidade de oxidação do $\mathrm{NADH}+\mathrm{H}^{+}$foi acompanhada a $340 \mathrm{~nm}$ em espectrofotômetro 
BECKMAN DU 640. O efeito do $\mathrm{pH}$ sobre o $\mathrm{K}_{\mathrm{M}}$ para o piruvato foi determinado sobre a fração $\mathrm{D} 70 \%$, na presença de diferentes concentrações de piruvato: 0,02 a 2,5 mM. A atividade enzimática foi determinada em tampão fosfato de sódio $0,1 \mathrm{M}, \mathrm{pH}$ 6,0, 7,0 e 8,0.

A medida da energia de ativação sobre a atividade da $\mathrm{LDH}$ da fração $\mathrm{D} 70 \%$, foi analisada em meio de reação saturante de piruvato e $\mathrm{NADH}+\mathrm{H}^{+}$. Como descrito anteriormente, a amostra de LDH foi pré-incubada nas seguintes temperaturas $10,15,20,25,30,35,40,45$ e $50^{\circ} \mathrm{C}$ durante 5 minutos, determinando-se a atividade enzimática após cada incubação nas temperaturas indicadas.

O cálculo da energia de ativação foi realizado segundo a equação de Arrhenius.

$$
\log K=-\frac{E a}{2,3 R} \frac{1}{T}+\log A
$$

onde, $\mathrm{K}=$ constante de velocidade específica da reação para uma dada temperatura; $\mathrm{Ea}=$ energia de ativação; $\mathrm{A}=$ constante particular de cada reação. Uma vez que a velocidade de determinada reação é proporcional a $\mathrm{K}$, assumiu-se como $\log \mathrm{K}$ o $\log$ da velocidade.

A estabilidade da LDH à temperatura foi determinada em meio de reação contendo NADH + $\mathrm{H}^{+} 0,14 \mathrm{mM}$ e piruvato de sódio $1 \mathrm{mM}$, em tampão fosfato de sódio $0,1 \mathrm{M}, \mathrm{pH} 7,4$, em volume final de $1,0 \mathrm{ml}$. Alíquotas da fração D70\% foram incubadas nas seguintes temperaturas: $5,20,30$ e $50^{\circ} \mathrm{C}$ por 5 , 10,20 e 30 minutos, determinando-se a atividade enzimática após cada incubação. A velocidade de oxidação do $\mathrm{NADH}^{+}+\mathrm{H}^{+}$foi acompanhada em espectrofotômetro BECKMAN DU 640, em comprimento de onda de $340 \mathrm{~nm}$, à temperatura de $25^{\circ} \mathrm{C}$ para Prochilodus scropha e entre $8,0 \pm 2,0^{\circ} \mathrm{C}$ para Notothenia neglecta.

$\mathrm{O}$ efeito da temperatura sobre o $\mathrm{K}_{\mathrm{M}}$ da LDH para o piruvato foi determinado sobre a fração D70\% frente a concentrações de piruvato de $0,02 \mathrm{mM}$ $2,5 \mathrm{mM}$. O meio de reação, contendo a amostra da LDH em diluições adequadas, foi mantido nas seguintes temperaturas, 10,20 e $30^{\circ} \mathrm{C}$ durante 5 minutos. A atividade enzimática foi determinada nessas temperaturas após cada incubação, com adição de piruvato de sódio nas concentrações indicadas.

\section{Resultados}

Análise do comportamento cinético da LDH de músculo estriado de Prochilodus scropha e Notothenia neglecta obtida a partir de purificação parcial foi realizada pelo estudo dos parâmetros que a seguir são tratados. Lactato desidrogenase de Prochilodus scropha e de Notothenia neglecta mostraram pH ótimo em torno de 6,5-7,5 e 6,6-7,0 respectivamente, usando-se para as determinações, tampão TRIS-HCl 0,2 M, tampão citrato-fosfato 0,1 $\mathrm{M}$, tampão fosfato $0,2 \mathrm{M}$, nos diferentes valores de $\mathrm{pH}$, de acordo com as Fig. 1 e 2.

Fig 1. Efeito do $\mathrm{pH}$ na atividade da lactato desidrogenase de músculo estriado de Prochilodus scropha. O meio de reação continha $\mathrm{NADH}+\mathrm{H}^{+} 0,14 \mathrm{mM}$ e piruvato de sódio $1,0 \mathrm{mM}$ nos seguintes tampões: tampão fosfato $0,2 \mathrm{M}$ em pH variando de 6,0 a 8,5, tampão TRIS-HCl 0,2 M em pH de 5,2 a 9,0 e tampão citrato-fosfato $0,1 \mathrm{M}$ em pH variando de 3,6 a 7,0. A reação foi iniciada pela adição de $10 \mu \mathrm{l}$ da preparação de $\mathrm{LDH}$ contendo $30,18 \mathrm{mg} / \mathrm{ml}$ de proteína. A velocidade da oxidação do $\mathrm{NADH}+\mathrm{H}^{+}$foi acompanhada a $340 \mathrm{~nm}$, em espectrofotômetro BECKMAN DU 640, à temperatura de $25 \pm 2,0^{\circ} \mathrm{C}$. 
Fig 2. Efeito do $\mathrm{pH}$ na atividade da lactato desidrogenase de músculo estriado de Notothenia neglecta. O meio de reação continha $\mathrm{NADH}+\mathrm{H}^{+} 0,14 \mathrm{mM}$ e piruvato de sódio $1,0 \mathrm{mM}$ nos seguintes tampões: tampão fosfato $0,2 \mathrm{M}$ em $\mathrm{pH}$ variando de 6,0 a 8,5, tampão TRIS-HCl 0,2 M em pH de 5,2 a 9,0 e tampão citrato-fosfato $0,1 \mathrm{M}$ em pH variando de 3,6 a 7,0. A reação foi iniciada pela adição de $10 \mu \mathrm{l}$ da preparação de $\mathrm{LDH}$, contendo $10,77 \mathrm{mg} / \mathrm{ml}$ de proteína. A velocidade da oxidação do $\mathrm{NADH}+\mathrm{H}^{+}$foi acompanhada a $340 \mathrm{~nm}$, em espectrofotômetro BECKMAN DU 640, à temperatura de $8,0 \pm 2,0^{\circ} \mathrm{C}$.

Com relação ao estudo da influência da temperatura sobre a atividade da LDH da fração D70\% verificou-se que a elevação da temperatura até $40{ }^{0} \mathrm{C}$ resultou em atividade máxima da enzima, porém, a $50{ }^{\circ} \mathrm{C}$, houve perda de $30 \%$ da atividade da enzima de Prochilodus scropha. A LDH de Notothenia neglecta, por sua vez apresentou atividade máxima em $30^{\circ} \mathrm{C}$ e perda de $50 \%$ da atividade em $50^{\circ} \mathrm{C}$. Os resultados obtidos foram registrados na forma de gráfico de Arrhenius e a energia de ativação foi de $7.591 \mathrm{cal} / \mathrm{mol}$ para Prochilodus scropha e de $6.567 \mathrm{cal} / \mathrm{mol}$ para Notothenia neglecta, a curva estabelecida tendo sido linear no intervalo de temperatura abrangido no experimento (Fig. $3)$.

Fig 3. Determinação da energia de ativação da LDH de músculo estriado de Prochilodus scropha e de Notothenia neglecta. A atividade da LDH foi medida em meio de reação contendo tampão fosfato $0,1 \mathrm{M}, \mathrm{pH} 7,4, \mathrm{NADH}+\mathrm{H}^{+} 0,14 \mathrm{mM}, 10 \mu 1 \mathrm{da}$ preparação de $\mathrm{LDH}$, contendo 15,40 e $10,67 \mathrm{mg} / \mathrm{ml}$ de proteína $/ \mathrm{ml}$, respectivamente para Prochilodus scropha e de Notothenia neglecta e incubado nas temperaturas de $10,15,20,25,30,35,40,45$ e $50^{\circ} \mathrm{C}$ por 5 minutos. A reação foi iniciada pela adição de piruvato de sódio $(50 \mathrm{mM})$ ao meio de reação na concentração final de $1,0 \mathrm{mM}$. A velocidade da reação foi seguida a $340 \mathrm{~nm}$ em espectrofotômetro BECKMAN DU 640, pela oxidação do $\mathrm{NADH}^{+}+\mathrm{H}^{+}$nas temperaturas indicadas. 
Os valores de $\mathrm{K}_{\mathrm{M}}$ com relação ao $\mathrm{pH}$, nas distintas situações são mostrados na Tab. 1 e indicam a relação que existe entre o pH em tampão fosfato e a velocidade a ação da LDH preparada de Prochilodus scropha e de Notothenia neglecta.
Os valores de $\mathrm{K}_{\mathrm{M}}$ com relação à temperatura são mostrados na Tab. 2 e indicam a relação que existe entre a temperatura e a velocidade de ação da LDH preparada de Prochilodus scropha e de Notothenia neglecta.

Tabela 1. Efeito do $\mathrm{pH}$ sobre o $\mathrm{K}_{\mathrm{M}}$ para piruvato da LDH de Prochilodus scropha e de Notothenia neglecta.. Os precipitados 70\% (P70\%) obtidos como descrito em Métodos foram dialisados contra tampão fosfato $10 \mathrm{mM}, \mathrm{pH} 6,5$, contendo $\beta \mathrm{ME} 1 \mathrm{mM}$ e glicerol $10 \%$, por aproximadamente 15 horas em câmara fria. As preparações, assim obtidas, foram centrifugadas a $10.000 \mathrm{rpm}$ durante $30 \mathrm{~min}$, em centrífuga refrigerada K70D. Os sobrenadantes (D70\%), contendo 10,67 e $15,40 \mathrm{mg} / \mathrm{ml}$ de proteína para Notothenia neglecta e Prochilodus scropha, respectivamente, foram utilizados para o presente estudo. Para as determinações de atividade foi utilizado sistema contendo tampão fosfato $0,1 \mathrm{M}, \mathrm{NADH}+\mathrm{H}^{+} 0,14 \mathrm{mM}, 10 \mu \mathrm{l}$ de LDH em diluições adequadas, piruvato de sódio 0,02-2,5 mM, em diferentes $\mathrm{pH} 6,0,7,0$ e 8,0.

\begin{tabular}{|c|c|c|c|c|c|c|}
\hline Constante & \multicolumn{3}{|c|}{ Prochilodus scropha } & \multicolumn{3}{|c|}{ Notothenia neglecta } \\
\hline $\mathrm{pH}$ & 6,0 & 7,0 & 8,0 & 6,0 & 7,0 & 8,0 \\
\hline $\mathrm{Vmax}(\mathrm{U} / \mathrm{ml})$ & $\begin{array}{r}371,30 \\
\pm 41,67\end{array}$ & $\begin{array}{r}373,26 \\
\pm 28,54\end{array}$ & $\begin{array}{c}483,24 \\
\pm 107,35\end{array}$ & $\begin{array}{l}262,01 \\
\pm 4,54\end{array}$ & $\begin{array}{l}342,01 \\
\pm 2,21\end{array}$ & $\begin{array}{r}313,39 \\
\pm 15,16\end{array}$ \\
\hline $\mathrm{K}_{\mathrm{M}}(\mathrm{mM})$ & $\begin{array}{r}0,048 \\
\pm 0,016 \\
\end{array}$ & $\begin{array}{r}0,046 \\
\pm 0,011 \\
\end{array}$ & $\begin{array}{r}0,144 \\
\pm 0,062 \\
\end{array}$ & $\begin{array}{c}0,044 \\
\pm 0,0034 \\
\end{array}$ & $\begin{array}{r}0,153 \\
\pm 0,0148 \\
\end{array}$ & $\begin{array}{r}0,277 \\
\pm 0,038 \\
\end{array}$ \\
\hline
\end{tabular}

Tabela 2. Efeito da temperatura sobre o $\mathrm{K}_{\mathrm{M}}$ para piruvato na atividade da LDH de Prochilodus scropha e de Notothenia neglecta. Os precipitados 70\% (P70\%) obtidos como descrito em Métodos foram dialisados contra tampão fosfato $10 \mathrm{mM}$, $\mathrm{pH}$ 6,5 contendo $\beta \mathrm{ME} 1 \mathrm{mM}$ e glicerol $10 \%$ por aproximadamente 15 horas em câmara fria. Os materiais assim obtidos foram centrifugados a $10.000 \mathrm{rpm}$ durantle $30 \mathrm{~min}$, em centrífuga refrigerada K70D. Os sobrenadantes (D70\%), contendo 10,67 e $15,40 \mathrm{mg} / \mathrm{ml}$ de proteína para Notothenia neglecta e Prochilodus scropha, respectivamente, foram utilizados para o estudo, em meio contendo tampão fosfato $0,1 \mathrm{M}, \mathrm{pH} 7,4, \mathrm{NADH}+\mathrm{H}^{+} 0,14 \mathrm{mM}, 10 \mu \mathrm{l}$ de $\mathrm{LDH}$ em diluições adequadas, piruvato de sódio 0,02-2,5 mM. O meio contendo $\mathrm{LDH}$ foi mantido durante 5 minutos nas diferentes temperaturas $\left(10,20 \mathrm{e} 30{ }^{\circ} \mathrm{C}\right)$. A reação foi iniciada com a adição do piruvato e a leitura em espectrofotômetro, a $340 \mathrm{~nm}$, nas temperaturas de estudo.

\begin{tabular}{|c|c|c|c|c|c|c|}
\hline \multirow[t]{3}{*}{ Parâmetros } & \multicolumn{3}{|c|}{ Prochilodus scropha } & \multicolumn{3}{|c|}{ Notothenia neglecta } \\
\hline & \multicolumn{6}{|c|}{ Temperatura $\left({ }^{\circ} \mathrm{C}\right)$} \\
\hline & 10 & 20 & 30 & 10 & 20 & 30 \\
\hline $\begin{array}{l}\mathrm{Vmax} \\
(\mathrm{U} / \mathrm{ml})\end{array}$ & $\begin{array}{r}953,39 \\
\pm 19,44\end{array}$ & $\begin{array}{r}1.423,05 \\
\pm 102,92\end{array}$ & $\begin{array}{l}1.403,82 \\
\pm 110,10\end{array}$ & $\begin{array}{r}569,39 \\
\pm 55,35\end{array}$ & $\begin{array}{r}858,15 \\
\pm 81,40\end{array}$ & $\begin{array}{r}975,06 \\
\pm 38,03\end{array}$ \\
\hline $\begin{array}{c}\mathrm{K}_{\mathrm{M}} \\
(\mathrm{mM})\end{array}$ & $\begin{array}{c}0,091 \\
\pm 0,006\end{array}$ & $\begin{array}{c}0,13 \\
\pm 0,023\end{array}$ & $\begin{array}{c}0,13 \\
\pm 0,029\end{array}$ & $\begin{array}{c}0,24 \\
\pm 0,054\end{array}$ & $\begin{array}{c}0,31 \\
\pm 0,062\end{array}$ & $\begin{array}{r}0,33 \\
\pm 0,035\end{array}$ \\
\hline
\end{tabular}

\section{Discussão}

Vários trabalhos são relatados na literatura com enzimas da via glicolítica em peixes antárticos SOMERO e HOCHACHKA, 1968; LOW e SOMERO, 1976; FITCH, 1988; BACILA et al., 1989; FELLER et al.., 1991; ZAMORA et al., 1992. Entretanto, estudos bioquímicos em peixes antárticos, com relação à lactato desidrogenase, são limitados a poucos trabalhos (FITCH, 1988;
BACILA et al., 1989 e FELLER et al., 1991; CARVALHO, 1997; CARVALHO et al., 1998).

BACILA et al. (1989), realizaram estudos sobre o metabolismo tecidual do "ice-fish" Chaenocephalus aceratus, estabelecendo os níveis das enzimas glicolíticas em músculo estriado, músculo cardíaco e encéfalo. Verificaram que o “ice-fish" possui níveis altos de PK, PFK, HK e em particular de LDH em todos os tecidos analizados. Estes mesmos autores verificaram que o "ice-fish" 
Chaenocephalus aceratus possui os níveis enzimáticos, inclusive os de LDH, superiores aos de outros peixes antárticos tais como Notothenia rossii e Notothenia neglecta.

Estes fatos mostram que um estudo mais aprofundado das enzimas da via glicolítica é de fundamental importância para melhor entender $\mathrm{o}$ comportamento bioquímico e fisiológico desses animais.

As diferenças na inibição pelo substrato têm sido sugeridas como a base para se distinguir o desempenho fisiológico de isoenzimas da $\mathrm{LDH}$ da maioria dos vertebrados. A LDH tipo $\mathrm{H}$ tem atividade máxima em baixas concentrações de piruvato sendo fortemente inibida em altas concentrações. O tipo $\mathrm{M}$ mantém sua atividade em altas concentrações de piruvato. A inibição da LDH do músculo cardíaco pelo piruvato favorece a completa oxidação de piruvato e lactato pela via oxidativa na mitocôndria, enquanto a LDH do músculo esquelético permite o suprimento rápido de energia via glicólise, convertendo o piruvato que se forma em lactato e liberando-o para o sangue, para ser metabolizado como fonte de energia pelo músculo cardíaco principalmente durante exercícios vigorosos, hipóxia ou durante a sua recuperação (DAWSON et al., 1964; MENDIOLA e DE COSTA, 1991). O mecanismo proposto para inibição da LDH pelo piruvato é a formação do complexo ternário abortivo, $\mathrm{LDH}_{-\mathrm{NAD}}{ }^{+}$piruvato, resultando na interação do $\mathrm{NAD}^{+}$com o piruvato e a enzima. A oxidação do $\mathrm{NADH}^{+}+\mathrm{H}^{+}$ para $\mathrm{NAD}^{+}$durante a redução catalisada pela LDH de piruvato para lactato resulta na formação do complexo ternário. Este complexo inibe competitivamente a redução de piruvato pelo $\mathrm{NADH}^{+}+\mathrm{H}^{+}$. A constante de dissociação para o complexo ternário da LDH do músculo cardíaco é menor do que para a LDH do músculo esquelético, com o resultado de que a $\mathrm{LDH}$ do coração é inibida em condições fisiológicas enquanto que a forma muscular esquelética tem significativa atividade.

As concentrações de substrato e de coenzima são fatores importantes no sistema da LDH. A LDH tem habilidade para formar complexos inativos ternários entre a enzima, a coenzima e o substrato, sendo a inibição da $\mathrm{LDH}$ o resultado deste complexo. Altas quantidades de $\mathrm{NADH}^{+}+\mathrm{H}^{+}$, induzem uma dissociação do complexo inativo causando reativação da enzima e oxidação do $\mathrm{NADH}^{+}+\mathrm{H}^{+} ;$o efeito contrário ocorre se a concentração de $\mathrm{NADH}^{+}+\mathrm{H}^{+}$diminuir, levando a formação do complexo inativo e portanto a um bloqueio da oxidação do $\mathrm{NADH}^{+}+\mathrm{H}^{+}$. O complexo ternário tem papel regulador no metabolismo do piruvato-lactato (FROMM, 1963; EVERSE et al.., 1971; NARITA e HORIUCHI, 1979; ARRIAGA et al., 1982 e HAMM, 1990).

Os efeitos da temperatura sobre a estrutura das enzimas e a sua função sempre foram do interesse de pesquisadores da área biológica. $\mathrm{Na}$ determinação do efeito da temperatura sobre a atividade da LDH do músculo de Prochilodus scropha esta apresentou-se estável em $5^{0} \mathrm{C}$ aumentando a atividade após 20 minutos de incubação e decrescendo sua atividade em $50{ }^{0} \mathrm{C}$ após 5 minutos. Em $30^{\circ} \mathrm{C}$ houve considerável aumento da atividade da $\mathrm{LDH}$ de aproximadamente $50 \%$. Lactato desidrogenase de Notothenia neglecta apresentou estabilidade em $5^{0} \mathrm{C}$, decrescendo sua atividade com o aumento da temperatura. $\mathrm{Na}$ temperatura de $20^{\circ} \mathrm{C}$ a $\mathrm{LDH}$ apresentou o dobro da atividade em relação a $5^{\circ} \mathrm{C}$ (Figs. 4 e 5 ).

Fig 4. Estabilidade da LDH de músculo estriado de Prochilodus scrpha em função da temperatura. O meio de reação (1ml) continha tampão fosfato $0,1 \mathrm{M}$, piruvato de sódio $1,0 \mathrm{mM}$, NADH $+\mathrm{H}^{+} 0,14 \mathrm{mM}$ em pH 7,4. A reação foi iniciada pela adição de $10 \mu \mathrm{l}$ da preparação de $\mathrm{LDH}$ pré-incubada às temperaturas de $5,20,30$ e $50^{\circ} \mathrm{C}$ por $5,10,20$ e 30 min. A velocidade da reação foi seguida a $340 \mathrm{~nm}$ em espectrofotômetro BECKMAN DU 640, pela oxidação do NADH $+\mathrm{H}^{+}$a $25,0 \pm 2,0^{\circ} \mathrm{C}$. 
Fig 5. Estabilidade da LDH de músculo estriado de Notothenia neglecta em função da temperatura. O meio de reação (1ml) continha tampão fosfato $0,1 \mathrm{M}$, piruvato de sódio $1,0 \mathrm{mM}, \mathrm{NADH}+\mathrm{H}^{+} 0,14 \mathrm{mM} \mathrm{em} \mathrm{pH} \mathrm{7,4.} \mathrm{A} \mathrm{reação} \mathrm{foi} \mathrm{iniciada} \mathrm{pela} \mathrm{adição}$ de $10 \mu \mathrm{l}$ da preparação de $\mathrm{LDH}$ pré-incubada às temperaturas de $5,20,30$ e $50^{\circ} \mathrm{C}$ por $5,10,20$ e 30 min. A velocidade da reação foi seguida a $340 \mathrm{~nm}$ em espectrofotômetro BECKMAN DU 640, pela oxidação do $\mathrm{NADH}+\mathrm{H}^{+}$a $8,0 \pm 2,0^{\circ} \mathrm{C}$.

GEREZ DE BURGOS et al. (1973; 1974), com músculo esquelético e cardíaco de cobra Bothrops neuwiedii e de bovino, verificaram que em diferentes concentrações de piruvato e temperatura, a atividade da LDH aumenta e chega à atividade máxima em pequenas concentrações de piruvato (0,01-0,05 $\mathrm{mM})$ a $10^{\circ} \mathrm{C}$ do que em alta temperatura, $35^{\circ} \mathrm{C}$. Para ambas as espécies estudadas, os valores de $\mathrm{K}_{\mathrm{M}}$ diminuiram com o decréscimo da temperatura, a redução tem sido maior para a LDH de Bothrops neuwiedii. Não houve inibição da atividade da LDH-A4 em altas concentrações de lactato $(200 \mathrm{mM})$. A $10^{\circ} \mathrm{C}$, a atividade com $200 \mathrm{mM}$ de lactato foi de $60 \%$ para a soenzima LDH-B4 de ambas as espécies e $77 \%$ para as isoenzimas de Bothrops neuwiedii e $80 \%$ para bovino em $35^{\circ} \mathrm{C}$. Os valores de $\mathrm{K}_{\mathrm{M}}$ para lactato de isoenzimas $\mathrm{B} 4$ para ambas as espécies não foram afetados pela temperatura.

SHAKLEE et al. (1977), analisaram a atividade da LDH de diferentes tecidos de Lepomis cyanellus "green sunfish" aclimatado em 5 e $25^{\circ} \mathrm{C}$. A atividade da LDH do músculo cardíaco, olho, cérebro e fígado permaneceu constante nas diferentes temperaturas. Entretanto, a atividade da LDH do músculo esquelético aumentou com a elevação da temperatura.

A energia de ativação para Prochilodus scropha e Notothenia neglecta foi medida entre 10 e $50^{\circ} \mathrm{C}$ e calculada de acordo com a equação de Arrhenius.

Outro fator a ser considerado é o efeito da concentração hidrogênionica. A LDH de Prochilodus scropha e de Notothenia neglecta mostra características peculiares a cada uma delas.
A atividade da LDH parcialmente purificada de Prochilodus scropha e Notothenia neglecta, com relação a diferentes $\mathrm{pH}$, foi máxima em torno de 7,5 e 6,5 a 7,0, respectivamente (Fig. 1 e 2). Estes resultados estão de acordo com o observado por THÉBAULT et al., (1981); SASAKI et al., (1989); ALMEIDA-VAL et al., (1991) e SASSAKI et al., (1994). O $\mathrm{K}_{\mathrm{M}}$ para piruvato em Prochilodus scropha aumentou com o aumento do $\mathrm{pH}$. O valor mínimo do $\mathrm{K}_{\mathrm{M}}$ foi determinado em $\mathrm{pH} 7,0$, a atividade máxima tendo sido em torno de 0,15-0,20 $\mathrm{mM}$ de piruvato, conforme experimentos observados por HOCHACHKA e LEWIS (1971); GEREZ DE BURGOS et al., (1973); WILSON (1977); DOUMEN et al., (1986) e MARTÍNEZ et al., (1994). Com relação ao $\mathrm{K}_{\mathrm{M}}$ para piruvato em Notothenia neglecta este decresceu consideravelmente com o aumento do $\mathrm{pH}$. Em pH 6,0 não houve inibição da $\mathrm{LDH}$ com crescentes concentrações de piruvato, a atividade da LDH tendo sido máxima com $0,75 \mathrm{mM}$ de piruvato em pH 7,0 e 8,0 .

HOCHACHKA (1965), analisou LDH de fígado e de músculo esquelético de "goldfish" Carassius carassius em pH 8,0, a velocidade da reação com o nível de substrato mostrou uma hipérbole para a LDH do fígado e músculo. Em pH 7,15, a velocidade da reação aumentou com o aumento de piruvato, mas a forma da curva não se alterou; em pH 9,05 e 10,15 a curva apresentou-se sigmoidal.

GEREZ DE BURGOS et al. (1973), compararam a LDH do músculo esquelético e cardíaco de cobra Bothrops neuwiedii com a de bovino (Sigma), em diferentes pHs 6.0, 7.4 e 8.0, o 
$\mathrm{K}_{\mathrm{M}}$ a velocidade de reação aumentando com a elevação do $\mathrm{pH}$ sendo que a LDH de Bothrops neuwiedii apresentou-se mais sensível; em temperatura constante, o $\mathrm{K}_{\mathrm{M}}$ para a $\mathrm{LDH}$ de Bothrops neuwiedii foi maior entre 7,4 e 8,0 do que entre 6,0 e 7,4. MENDIOLA e DE COSTA (1991), verificaram o efeito do $\mathrm{pH}(7,0,7,4$ e 8,0$) \mathrm{em}$ função da temperatura $\left(15,20\right.$ e $\left.30^{\circ} \mathrm{C}\right)$ na $\mathrm{LDH}$ do músculo cardíaco de Rana perezi e Bufo calamita de diferentes comportamentos e habitats. $\mathrm{O} \mathrm{K}_{\mathrm{M}}$ para piruvato de ambas as espécies aumentou com o $\mathrm{pH}$ e com a temperatura, sendo que a velocidade máxima variou somente com a temperatura. Com relação ao $\mathrm{K}_{\mathrm{M}}$ para lactato este diferiu com a temperatura e variação do $\mathrm{pH}$ para ambas as espécies. $\mathrm{O} \mathrm{K}_{\mathrm{M}}$ para lactato de Rana perezi aumentou com relação à temperatura mas decresceu com relação à elevação do $\mathrm{pH}$ enquanto que $B u f o$ calamita não variou. A velocidade máxima em Rana perezi aumentou com a temperatura e em Bufo calamita a velocidade máxima variou com a temperatura e com o $\mathrm{pH}$.

COPPES et al., (1992), verificaram o efeito da temperatura e de $\mathrm{pH}$ sobre a LDH do músculo esquelético branco de três espécies da família Scianidae, Micropogonias furnieri, Cynoscion striatus e Roncador stearnsii. $\mathrm{O} \mathrm{K}_{\mathrm{M}}$ para piruvato permaneceu constante em $\mathrm{pH} 6,3$ a 7,2 nas temperaturas estudadas $\left(20,25\right.$ e $\left.30^{\circ} \mathrm{C}\right)$. Entretanto com o aumento do $\mathrm{pH}(7,6$ e 8,3$)$ os valores de $\mathrm{K}_{\mathrm{M}}$ aumentam cerca de oito vezes, nas diferentes temperaturas, para as espécies analisadas.

MARTÍNEZ et al., (1994), estudaram a LDH do músculo branco de duas espécies de peixes euritérmicos Cynoscion arenarius e Cynoscion nebulosus. $\mathrm{O} \mathrm{K}_{\mathrm{M}}$ para piruvato na espécie Cynoscion nebulosus aumentou com a elevação do $\mathrm{pH}$ e da temperatura enquanto que para Cynoscion arenarius permaneceu constante em $\mathrm{pH} 6,3$ e 6,8 nas diferentes temperaturas $\left(15,20,25\right.$ e $\left.30^{\circ} \mathrm{C}\right)$, aumentando consideravelmente em $\mathrm{pH} 7,6$, nas temperaturas acima de $15{ }^{\circ} \mathrm{C}$.

\section{REFERÊNCIAS BIBLIOGRÁFICAS}

ALMEIDA-VAL， V.M.F.; SCHWANTES， M.L.B.; VAL, A.L. LDH Isozymes in Amazon fish. II.Temperature and $\mathrm{pH}$ effects on LDH kinetic properties from Mylossoma duriventris and Colossoma macropomum (Serrasalmidae); Comp. Biochem. Physiol. 98B(1): 79-86, 1991.

ARRIAGA, D.; SOLER, J.; CADENAS, E. Influence of $\mathrm{pH}$ on the allosteric properties of lactate dehydrogenase activity of Pleycomyces blakesleeanus. Biochem. J. 203:393-400, 1982.

BACILA, M.; ROSA, R.; RODRIGUES, E.; LUCHIARI, P.H.; ROSA, C.D. Tissue metabolism of the ice-fish Chaenocephalus aceratus LOENBERG. Comp. Biochem. Physiol. 92B(2):313-318, 1989.
BAILEY, G.S.; WILSON, A.C. Homologies between isoenzymes of fishes and those of higher vertebrates. J. Biol. Chem. 243(22):5843-5853, 1968.

BERGMEYER, H.V.; BERN, T.E. Lactate desidrogenase U.V. assay with pyruvate kinase and LDH. In "Bergmeyer H.V., Ed. Methods of Enzymatic Analysis. $2^{\text {nd }}$ ed. New York, Verlag-Chemie, 1974.

CARVALHO, C.S. Purificação, caracterísitcas cinéticas e isoenzimas da lactato desidrogenase (L-lactato $\mathrm{NAD}^{+}$ oxidorreductase, E.C.1.1.1.27) do músculo epaxial de curimbatá Prochilodus scrofa (Steindachner, 1881) e de Notothenia neglecta (Pisces, Teleostei). Tese de mestrado em Fisiopatologia e Comportamento de Organismos Aquáticos, UFPR, 1997.

CARVALHO, C.S.; ROSA, R.; SASSAKI, K.T.; BACILA, M. Purificação e isoenzimas da lactato desidrogenase do músculo epaxial de Prochilodus scropha e Notothenia neglecta. Arch. Vet. Scienc. 3 (1): 65-72, 1998.

COPPES, Z.; MARTÍNEZ, G.; HIRSCHHORN, M. pH and Temperature effects on the $\mathrm{Km}$ values of muscle Lactate Dehydrogenase isozyme $\mathrm{LDH}-\mathrm{A}_{4}$ from fishes of the family Scianidae (Perciformes); Comp. Biochem. Physiol. 103B(4):869-874, 1992.

DAWSON, D.M.; GOODFRIEND, T.L.; KAPLAN, N.O. Lactic dehydrogenases: functions of the two types. Rates of synthesis of the two major forms can be correlated with metabolic differentiation. Science. 207:769-770, 1964.

DE WITT, H.H. Coastal and deep-water benthic fishes of the Antarctic. Am. Geogr. Soc. Antarct. Map Folio Ser. 15:1-10, 1971.

DOUMEN, C.; D'SUZE, G.; VERHEYEN, E.; BLUST, R. Temperature and ph effects on the total white muscle LDH of Oreochromis niloticus (Pisces; Cichlidae); Comp. Biochem. Physiol. 83B(2): 441-444, 1986.

EVERSE, J.; BARNETT, R.E.; THORNE, C.J.R.; KAPLAN, N.O. The formation of ternary complexes by diphosphopyridine nucleotide-dependent dehydrogenases. Arch. Biochem. Biophys. 143:444-460, 1971.

FELLER, G.; PAULY, J.P.; O'CARRA, P.; GERDAY, C. The lactate dehydrogenase of the icefish heart: biochemical adaptations to hypoxia tolerance. Biochim. Biophys. Acta. 1079, 3:343-347, 1991.

FITCH, N.A. Lactate dehydrogenases in Antarctic and temperate fish species. Comp. Biochem. Physiol. 91B, 4: 671-676, 1988 .

FROMM, H.J. Determination of dissociation constants of coenzymes and abortive ternary complexes with rabbit muscle lactate dehydrogenase from fluorescence measurements. J. Biol. Chem. 238, 9:2938-2944, 1963.

GEREZ DE BURGOS, N.M.; BURGOS, C.; GUTIERREZ, M.; BLANCO, A. Effect of temperature upon catalytic properties of lactate dehydrogenase isoenzymes from a poikilotherm. Biochim. Biophys. Acta. 315:250-258, 1973.

GEREZ DE BURGOS, N.M.; BURGOS, C.; BLANCO, A. Effect of temperature upon inhibition by substrate of lactate dehydrogenase isoenzymes from a poikilotherm. Biochim. Biophys. Acta. 341:505-510, 1974.

HAMM, R. Lactatdehydrogenase und ihre bedeutung in der fleischforschung. Fleischwirtschaft. 70, 11: 13361339, 1990. 
HECK, H. d'A. Porcine heart lactate dehydrogenase optical rotatory dispersion, thermodynamics, and kinetics of binding reactions. J. Biol. Chem. 244:4375-4381, 1969.

HOCHACHKA, P.W. Isozymes in metabolic adaptation of a poikilotherm: subunit relationships in lactic dehydrogenases of goldfish; Arch. Biochem. Biophys. 111:96-103, 1965.

HOCHACHKA, P.W.; SOMERO, G.N. The adaptation of enzymes to temperature. Comp. Biochem. Physiol.. 27: 659-668, 1968.

HOCHACHKA, P.W.; LEWIS, J.K. Interacting effects of $\mathrm{pH}$ and temperature on the $\mathrm{Km}$ values for fish tissue lactate dehydrogenases. Comp. Biochem. Physiol. 39B:925-933, 1971.

KLYACHKO, O.S.; OZERNYUK, N.D. The effect of temperature on the kinectic properties of lactate dehydrogenase from embryos of various fish species. Comp. Biochem. Physiol. 107B(4):593-595, 1994.

LOW, P.; SOMERO, G. Adaptation of muscle pyruvate kinase to environmental temperatures and pressures. J. Exp. Zoll. 198:1-11, 1976.

LOWRY, D.R.; ROSEBROUGH, N.J.; FARR, A.L.; RANDALL, R.J. Protein measurement with the folin phenol reagent. J. Biol. Chem. 193:265-275, 1951.

MARTINEZ, G.; BEHRENS, P.; COPPES, Z. pH and temperature influences on the $\mathrm{Km}$ values of LDH-A4 from white muscles of two eurythermal Scianidae fishes. Comp. Biochem. Physiol. 107B(4):645-648, 1994.

MENDIOLA, P.; DE COSTA, J. The effects of temperature and $\mathrm{pH}$ on the kinetic properties of heart muscle lactate dehydrogenase from anuran amphibians. Comp. Biochem. Physiol. 98B(4): 529-534, 1991.

NARITA, J.I.; HORIUCHI, S. Effect of environmental temperature upon muscle lactate dehydrogenase in the crayfish, Procambarus clarki GIRARD. Comp. Biochem. Physiol. 64B:249-253, 1979.

OZERNYUK, N.D.; KLYACHKO, O.S.; POLOSUKHINA, E.S. Aclimation temperature affects the functional and structural properties of lactate dehydrogenase from fish (Misgurnus fossilis) skeletal muscles. Comp. Biochem. Physiol., 107 B(1):141-145, 1994.

REHSE, P.H.; DAVIDSON, W.S. Purification and properties of a C-type isozyme of lactate dehydrogenase from the liver of the Atlantic cod (Gadus morhua). Comp. Biochem. Physiol. 84B:145-150, 1985.

RODRIGUES, E.; ROSA, R.; BACILA, M. The effect of temperature on the hinetic properties of lactate dehudrogenase from the epaxial muscle of the Antarctic fish. Notothenia gibberifrons Lonnberg. Arq. Biol. Tecnol. 38(4):1231-1236, 1995.

SASAKI, T.; HYODO-TAGUCHI, Y.; IUCHI, I.; YAMAGAMI, K. Purification and partial characterization of the muscle LDH-A4 and -B4 isozymes and the respective subunits of the fish, Oryzias latipes. Comp. Biochem. Physiol. 93B1:11-20, 1989.

SASSAKI, K.T.; ROSA, C.D.; CERQUEIRA CÉSAR, M.; ROSA, R. Kinectic aspects of rat (Rattus norvegicus albinus) submandibular glands lactic dehydrogenase. Arq. Biol. Tecnol. 37, 2:333-343, 1994.
SHAKLEE, J.B.; CHRISTIANSEN, J.A.; SIDELL, B.D.; PROSSER, C.L.; WHITT, G.S.; Molecular aspects of temperature acclimation in fish: contributions of changes in enzyme activities and isozyme patterns to metabolic reorganization in the green sunfish. J. Exp. Zool. 201:1-20, 1977.

SIDELL, B.D.; BELAND, K.F. Lactate dehydrogenase of Atlantic hagfish: Physiological and evolutionary implications of a primitive heart isozyme. Science. 207:769-770, 1980.

SOMERO, G.N. pH-temperature interactions on proteins: principles of optimal $\mathrm{pH}$ and buffer system design. Mar. Biol. Letters. 2:163-178, 1981.

SOMERO, G.N.; HOCHACHKA, P.W. The effect of temperature on catalytic and regulatory functions of pyruvate kinase of the rainbow trout and the Antarctic fish Trematomus bernachii. Biochem. J. 110:395-400, 1968.

SPIELMANN, H.; ERICKSON, R.P.; EPSTEIN, C.J. The separation of lactate dehydrogenase $\mathrm{x}$ from other lactate dehydrogenase isozymes of mouse testes by affinity chromatography. FEBS.Lett. 35, 1:19-23, 1973.

STRAUB, F.B. Crystalline lactic dehydrogenase from heart muscle. Biochem. J. 34:483-486, 1940.

THÉBAULT, M.T.; BERNICARD, A.; LENNON, J.F. Lactate dehydrogenase from the caudal muscle of the shrimp Palaemon serratus: Purification and characterization. Comp. Biochem. Physiol. 68B:6570, 1981.

TSUKUDA, H.; Temperature dependency of the relative activities of liver lactate dehydrogenase isozymes in goldfish acclimated to different temperatures. Comp. Biochem. Physiol. 52B:343-345, 1975.

VESELL, E.S. pH Dependence of lactate dehydrogenase isozyme inhibition by substrate. Nature. 503423 : 421-422, 1966.

VESELL, E.S.; YIELDING, L. Effects of $\mathrm{pH}$, ionic strenghth, and metabolic intermediates on the rates of heat inactivation of lactate dehydrogenase isozymes. Proc. N.A.S. 56:1317-1324, 1966.

WILSON, T.L. Interrelations between $\mathrm{pH}$ and temperature for the catalytic rate of the $M_{4}$ isozyme of lactate dehydrogenase (E.C. 1.1..1.27) from goldfish (Carassius auratus L.). Arch. Biochim. Biophys. 79:378-390, 1977.

YANCEY, P.H.; SOMERO, G.N. Temperature dependence of intracellular $\mathrm{pH}$ : Its role in the conservation of pyruvate apparent $\mathrm{Km}$ values of vertebrate lactate dehydrogenases. J. Comp. Physiol. 125:129-134, 1978.

ZAMORA, J.M.; ROSA, R.; ROSA, C.D.; BIANCONCINI, M.S.C.; BACILA, M. Purification and properties of pyruvate kinase from the striated muscle of the ice-fish Chaenocephalus aceratus Loenberg.; Int. J. Biochem. 24, 11:1833-1840, 1992. 